\title{
Changes in Prevalence and Treatment Pattern of Benign Prostatic Hyperplasia in Korea
}

\author{
Jung Ki Jo ${ }^{1}$, Sung Ho Shinn ${ }^{2}$, Kyu Shik Kim ${ }^{1}$, Hong Sang Moon ${ }^{1}$ \\ ${ }^{1}$ Department of Urology, Hanyang University College of Medicine, Seoul, Korea \\ ${ }^{2}$ Department of Thoracic and Cardiovascular Surgery, Cheju Halla General Hospital, Jeju, Korea
}

\begin{abstract}
Purpose: Benign prostatic hyperplasia (BPH) is associated with lower urinary tract symptoms and negatively affects the quality of life. We aimed to investigate the treatment pattern of BPH in South Korea.

Methods: Information on treatment modalities and diagnoses of $\mathrm{BPH}$ was obtained from the Health Insurance Review and Assessment Service-Aged Patient Sample. Data on BPH patients aged $\geq 60$ years from 2012 to 2016 were obtained. We surveyed the treatment pattern of $\mathrm{BPH}$, including the types of drugs used and surgeries performed, according to the type of institution.

Results: In this study, 18,260-24,657 BPH patients treated between 2012 and 2016 were included. The number of patients showed an increasing pattern, and drug therapy was the major treatment method used for BPH (98.77\%). Moreover, the pattern of increased pharmacotherapy use for BPH was reinforced by the increasing number of patients. Prescription of a-blockers only was dominant in this cohort (45.7\%). Transurethral resection of the prostate (TURP) was the most commonly used surgical treatment for $\mathrm{BPH}$ (53.6\%), but it showed a decreasing pattern over time. In contrast, holmium laser enucleation of the prostate (HoLEP) showed an increase from $19.4 \%$ to $39.7 \%$.

Conclusions: The most common treatment for BPH was drug therapy, predominantly only $a$-blocker therapy. The surgical treatment trend has changed from TURP to HoLEP.
\end{abstract}

Keywords: Prostate; Therapeutics; Prevalence

- Grant/Fund Support: This study was funded by the Korean Continence Society.

- Research Ethics: We performed this study after approval from the Institutional Review Board of Hanyang University Hospital (HY-2020-07-022).

- Conflict of Interest: No potential conflict of interest relevant to this article was reported.

\section{INTRODUCTION}

Over $50 \%$ men aged $\geq 60$ years develop lower urinary tract symptoms (LUTS) [1,2]. Benign prostatic hyperplasia (BPH) is one of the most common benign conditions associated with reduced quality of life $[3,4]$. It may present as conditions such as LUTS, acute urinary retention (AUR), urinary tract infections, and hematuria [5]. BPH increases the risk of mortality and morbidity, and the cost of $\mathrm{BPH}$ treatment is high.

The etiology of BPH is unclear, and the known risk factors include old age and hormones including androgen and estrogen [6]. Multiple factors are associated with this benign condition in old age. Jeong et al. [7] have reported that patients with severe LUTS are at increased risk of depression, especially nocturia, which is a risk factor associated with depression. In our previous study, the severity of $\mathrm{BPH}$ increased with age, and the

Corresponding author: Hong Sang Moon (iD https://orcid.org/0000-0003-2101-1019 Department of Urology, Hanyang University Guri Hospital, 153 Gyeongchun-ro, Guri 11923, Korea

Email: moonuro@hanyang.ac.kr

Submitted: November 2, 2020 / Accepted after revision: December 21, 2020 
treatment pattern in $\mathrm{BPH}$ patients varied according to the socioeconomic status [8]. Several other factors, such as physical activity, body mass index, stress level, and smoking history have been associated with the severity of LUTS [9].

The treatment for $\mathrm{BPH}$ is diverse, ranging from drug therapy to a combination of both drug therapy and surgical treatment. However, there are limited studies on the prevalence and treatment pattern of BPH. Further, an optimal treatment plan is necessary in some patients to achieve the best clinical outcome. Therefore, we conducted a cross-sectional study on the prevalence and treatment pattern, including the types of drugs used and surgeries performed, of $\mathrm{BPH}$ using nationwide data.

\section{MATERIALS AND METHODS}

\section{Study Cohort}

We performed a cross-sectional study about BPH treatment procedures using nationwide data sample after approval from the Institutional Review Board of Hanyang University Hospital (HY-2020-07-022). We obtained information on BPH treatment trends from the Health Insurance Review and Assessment Service-Aged Patient Sample (HIRA-APS). We used data on men aged $\geq 60$ years from 2012 to 2016 from the database. We divided the cohort into 3 groups according to age $-60-69$ years, 70-79 years, and $\geq 80$ years. Our study analyzed the regional distribution of $\mathrm{BPH}$ population according to the year.

\section{Patient group setting (selected according to the following criteria)}

(1) Patients with BPH code N40 more than once within a year

(2) Patients who underwent urology treatment

(3) Age $\geq 60$ years (excluded condition: $<60$ years)

Code N40: Hyperplasia of prostate without complication (N400); Adenofibromatous hypertrophy of prostate without complication (N400.01); Enlargement (benign) of prostate hyperplasia (N400.02); Hypertrophy (benign) of prostate without complication (N400.03); Median bar (prostate) of prostate without complication (N400.04); and Prostatic obstruction of prostate NOS without complication (N400.05)

\section{Baseline characteristics}

(1) Age (60-69 years, $70-79$ years, and $\geq 80$ years)

(2) Area: This refers to location of the billing/nursing institution where the patient was treated. It may not match the location of the patient's residence.
(3) Type (hospital-level such as clinics and general hospitals or higher)

\section{Evaluating treatment trends by year}

Based on the prescription at the time of treatment of BPH code N40

(1) Evaluating the trends of surgery, pharmacotherapy, and surgery + pharmacotherapy by year to treat $\mathrm{BPH}$

(2) Identifying the type of surgery performed and drugs used by year to treat $\mathrm{BPH}$

\section{Analyzing prescription patterns}

(1) Evaluating the pattern of the drugs prescribed in the same specification, based on the drug recorded in the invoice when the patient with of $\mathrm{BPH}$ code $\mathrm{N} 40$ was treated at the urology department

(2) Checking the overall prescription patterns and by hospital type

\section{Primary Outcome}

We classified the type of institution and other variables using HIRA-APS codes.

The treatment pattern of $\mathrm{BPH}$ was evaluated according to the type of institution. The institutions were classified as primary clinics (hospitals, including clinics) and secondary and tertiary centers. We analyzed the medication and operative trends of $\mathrm{BPH}$. The medication trends were categorized as the 5 - $\alpha$-reductase inhibitor (5-ARI); 5-ARI and $\alpha$-blocker; 5-ARI, $\alpha$-blocker, and anticholinergics; and anticholinergics and $\alpha$-blocker groups.

The operative trends were categorized as the holmium laser enucleation of the prostate (HoLEP), transurethral resection of the prostate (TURP), GreenLight laser, and prostatectomy groups.

The data were analyzed using SAS 9.4 (SAS Institute Inc., Cary, NC, USA) as mentioned above.

\section{RESULTS}

\section{Basic Characteristics}

Data on the 18,260-24,657 men included in this cohort from 2012 to 2016 were obtained from HIRA-APS. The number of patients increased from 20,114 to 27,390 from 2012 to 2016 (Table 1). Regional distribution of BPH population showed a similar pattern every year (Fig. 1). 
Table 1. Total cohort and treatment trend for benign prostatic hyperplasia

\begin{tabular}{|c|c|c|c|c|c|}
\hline \multirow{2}{*}{ Variable } & \multicolumn{5}{|c|}{ Year } \\
\hline & 2012 & 2013 & 2014 & 2015 & 2016 \\
\hline \multicolumn{6}{|l|}{ Age (yr) } \\
\hline $60-69$ & $9,407(46.8)$ & $10,126(46)$ & $10,663(45.4)$ & $11,309(44.5)$ & $12,050(44)$ \\
\hline $70-79$ & $8,208(40.8)$ & $9,104(41.3)$ & $9,591(40.8)$ & $10,349(40.7)$ & $11,176(40.8)$ \\
\hline$\geq 80$ & $2,499(12.4)$ & $2,798(12.7)$ & $3,252(13.8)$ & $3,782(14.9)$ & $4,164(15.2)$ \\
\hline Total & $20,114(100)$ & $22,028(100)$ & $23,506(100)$ & $25,440(100)$ & $27,390(100)$ \\
\hline \multicolumn{6}{|l|}{ Treatment trend } \\
\hline Surgery & $22(0.12)$ & $17(0.08)$ & $11(0.05)$ & $9(0.04)$ & $17(0.07)$ \\
\hline Surgery+drug & $287(1.57)$ & $276(1.38)$ & $302(1.41)$ & $312(1.36)$ & $286(1.16)$ \\
\hline Drug & $17,951(98.31)$ & $19,736(98.54)$ & $21,064(98.54)$ & $22,652(98.6)$ & $24,354(98.77)$ \\
\hline Total & $18,260(100)$ & $20,029(100)$ & $21,377(100)$ & $22,973(100)$ & $24,657(100)$ \\
\hline \multicolumn{6}{|c|}{ Hospital including clinic } \\
\hline Surgery & $6(0.05)$ & $5(0.04)$ & $3(0.02)$ & $1(0.01)$ & $6(0.04)$ \\
\hline Surgery+drug & $146(1.28)$ & $141(1.13)$ & $148(1.11)$ & $135(0.95)$ & $135(0.88)$ \\
\hline Drug & $11,232(98.66)$ & $12,367(98.83)$ & $13,169(98.87)$ & $14,066(99.04)$ & $15,213(99.08)$ \\
\hline Total & $11,384(100)$ & $12,513(100)$ & $13,320(100)$ & $14,202(100)$ & $15,354(100)$ \\
\hline \multicolumn{6}{|c|}{ Secondary and tertiary center } \\
\hline Surgery & $21(0.24)$ & $16(0.17)$ & $10(0.1)$ & $9(0.08)$ & $14(0.12)$ \\
\hline Surgery+drug & $221(2.57)$ & $228(2.44)$ & $259(2.6)$ & $275(2.56)$ & $255(2.22)$ \\
\hline Drug & $8,355(97.19)$ & $9,104(97.39)$ & $9,678(97.3)$ & $10,467(97.36)$ & $11,193(97.65)$ \\
\hline Total & $8,597(100)$ & $9,348(100)$ & $9,947(100)$ & $10,751(100)$ & $11,462(100)$ \\
\hline
\end{tabular}

Values are presented as number (\%).

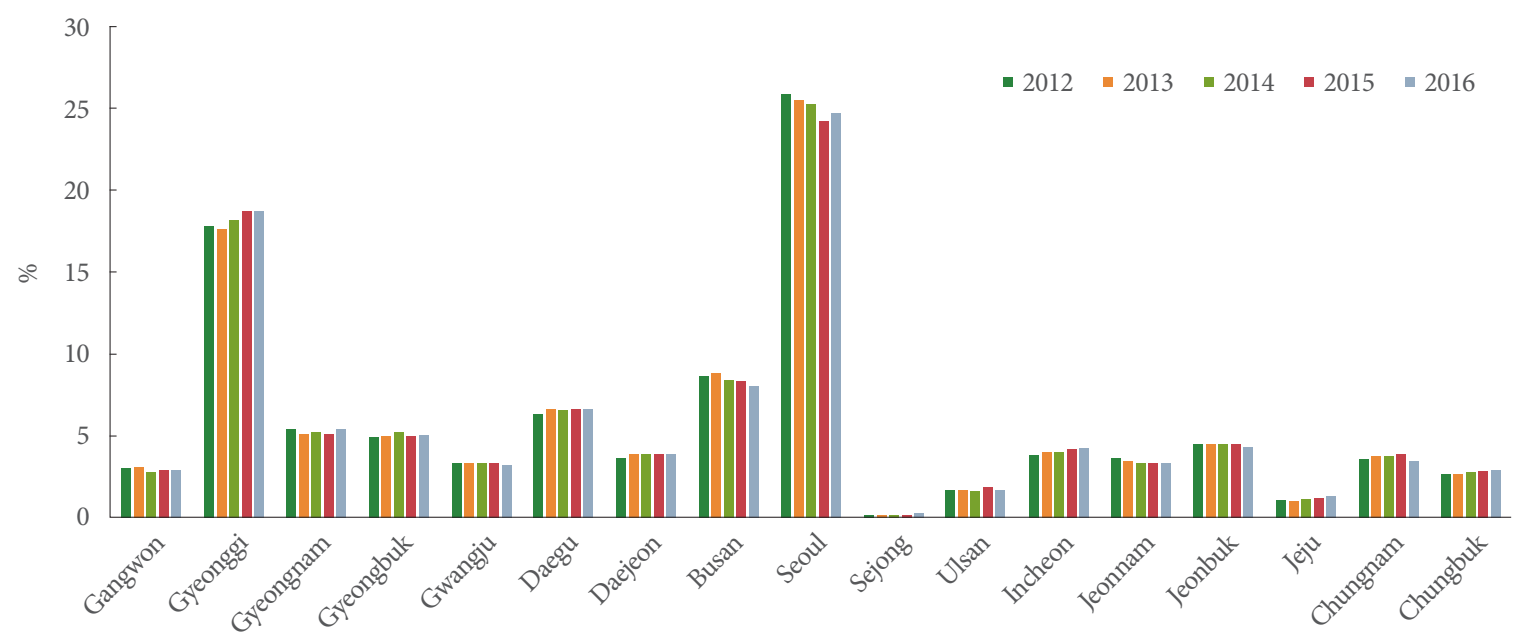

Fig. 1. Regional distribution of benign prostatic hyperplasia population in South Korea according to years.

\section{Treatment Trends of BPH}

The number of BPH patients who were treated at clinics and tertiary centers showed an increasing trend every year. This pat- tern was observed for all treatment methods, including surgery and combination treatment. Treatment with medications was the most commonly used treatment option in $>90 \%$ cases. As 


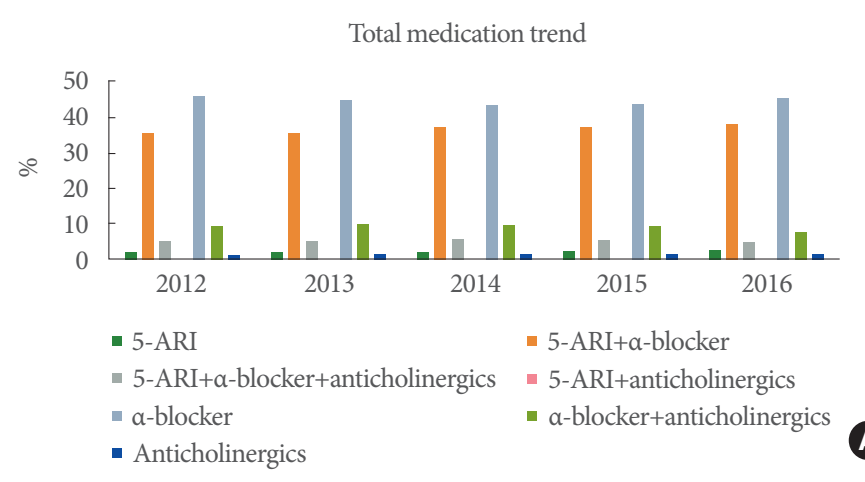

Hospotal including clinic medication trend

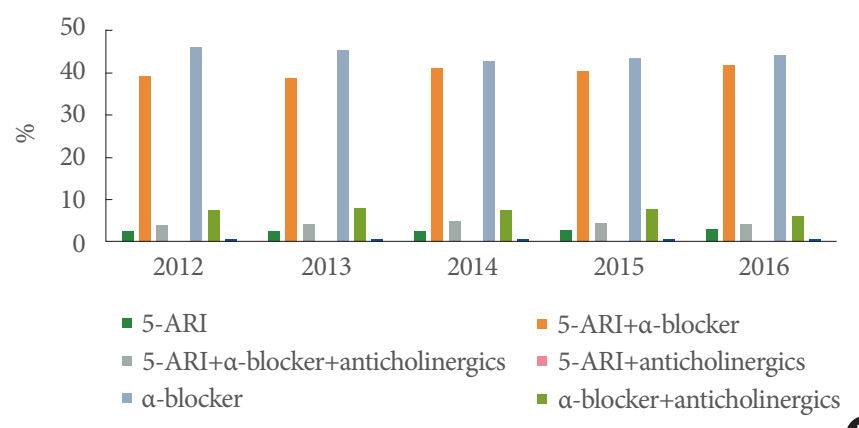

B

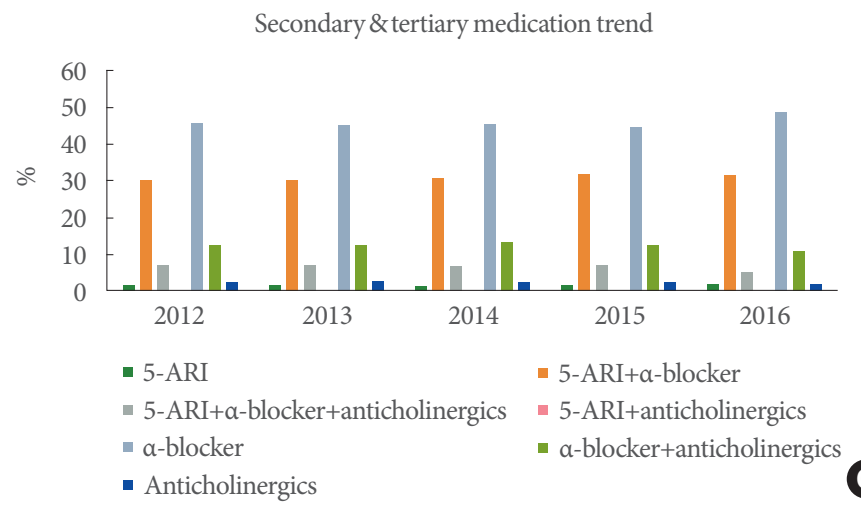

Fig. 2. (A) Total medication trend for benign prostatic hyperplasia (BPH) according to years. (B) Hospital (including clinic) medication trend for BPH according to years. (C) Secondary and tertiary medication trend for BPH according to years. 5-ARI, 5- $\alpha$-reductase inhibitor.

the number of patients increased, the medication usage trend also increased. Moreover, both surgery and combination treatment showed an increasing pattern.

\section{Medication Treatment Trend}

Alpha-blockers were the most common medication recommended for BPH treatment, followed by a combination therapy
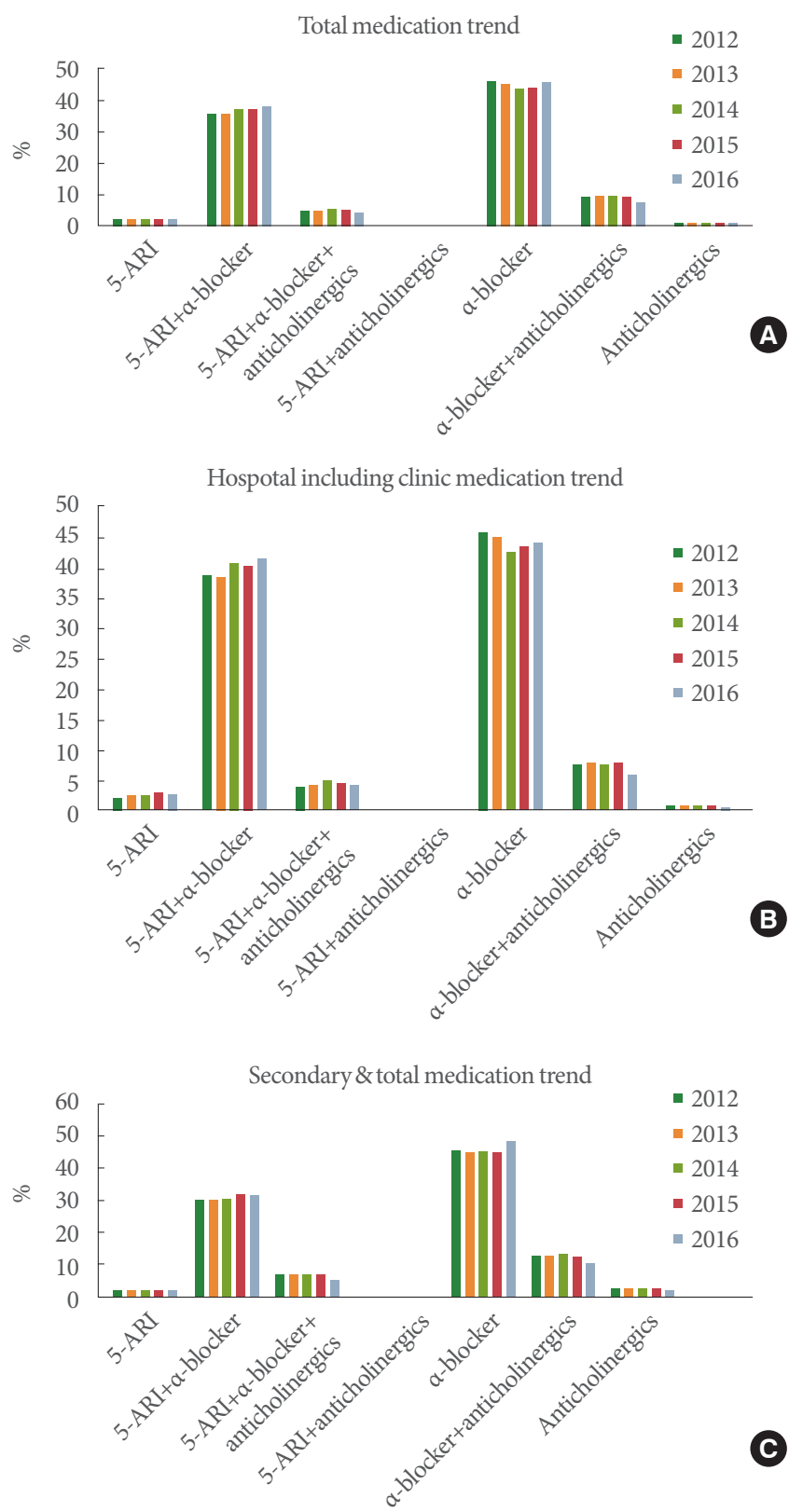

Fig. 3. (A) Total medication trend for benign prostatic hyperplasia (BPH) according to kind of drugs. (B) Hospital (including clinic) medication trend for $\mathrm{BPH}$ according to kind of drugs. (C) Secondary and tertiary medication trend for $\mathrm{BPH}$ according to kind of drugs. 5-ARI, 5- $\alpha$-reductase inhibitor.

of $\alpha$-blocker and 5-ARI. A similar pattern (combination pattern such as $\alpha$-blocker and 5-ARI) was observed in both clinics and tertiary centers throughout the study period (Fig. 2). Anticholinergics also used a combination therapy, but it was not ordinary use monotherapy. Medication trends of $\alpha$-blockers and combination therapies are shown in Fig. 3. 

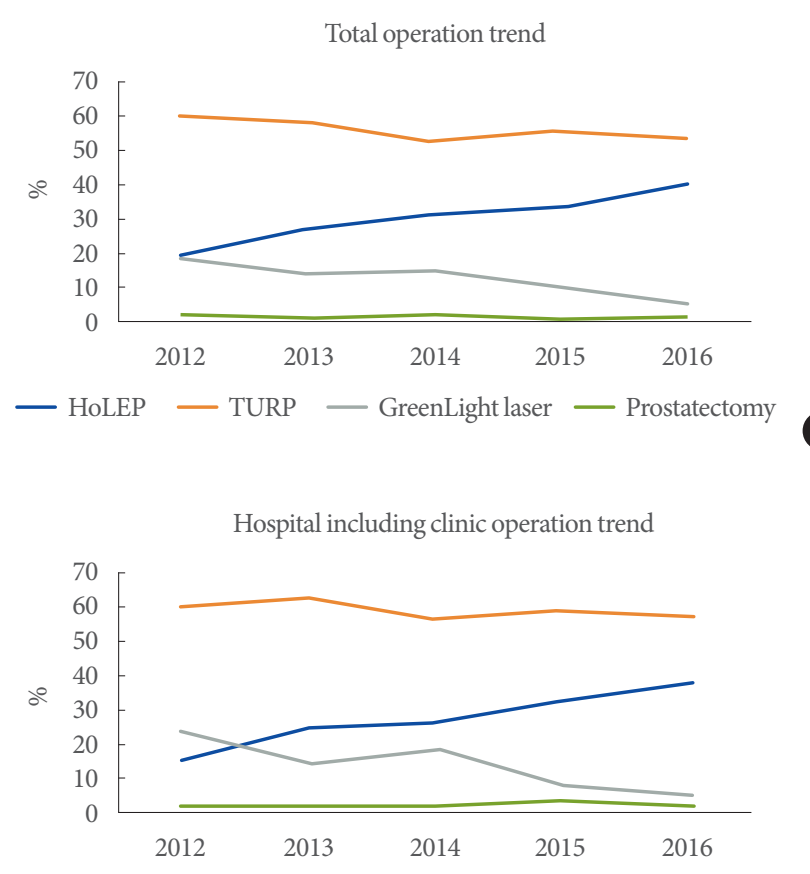

— HoLEP — TURP — GreenLight laser — Prostatectomy

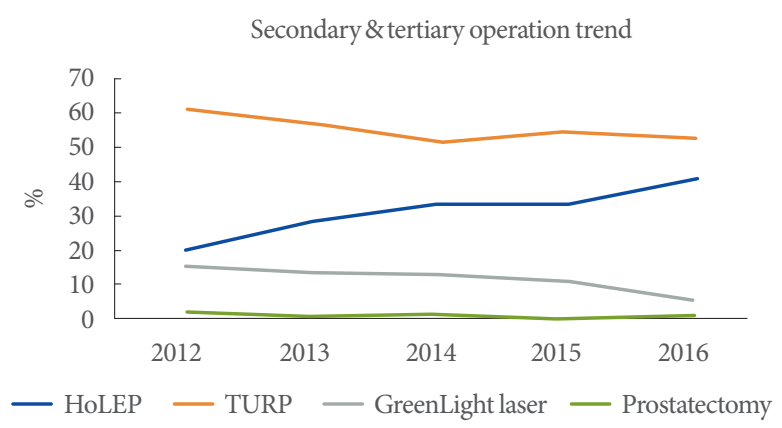

Fig. 4. (A) Total operation trend for benign prostatic hyperplasia (BPH) according to years. (B) Hospital (including clinic) operation trend for $\mathrm{BPH}$ according to years. (C) Secondary and tertiary operation trend for BPH according to years. HoLEP, holmium laser enucleation of the prostate; TURP, transurethral resection of the prostate.

\section{Surgical Treatment Trend}

TURP was the most common surgical method for $\mathrm{BPH}$ treatment $(60.2 \%$ in $2012 ; 57.9 \%$ in $2013 ; 54.7 \%$ in $2014 ; 56.0 \%$ in 2015; and 53.1\% in 2016) (Fig. 4). However, TURP and greenlight laser treatments showed a decreasing pattern, while HoLEP showed an increasing pattern over the study period $(20.5 \%$ in $2012 ; 25.6 \%$ in $2013 ; 29.5 \%$ in $2014 ; 33.5 \%$ in 2015 ; and $40.2 \%$ in 2016). This pattern was similar in both clinics and tertiary centers during the study period.

\section{DISCUSSION}

We analyzed the treatment pattern of BPH in South Korea using nationwide data. The number of BPH cases showed an accelerated increase in the superaged society. Although drug therapy was the common choice of treatment, surgical treatments showed an increasing pattern in our study. HoLEP showed an increasing pattern with the spread of the holmium laser use in South Korea.

Souverein et al. [10] analyzed the trend of BPH medications use from the PHARMO Record Linkage System. Their data showed that there was an increase in its use from 1.9/1,000 in 1,991 to $25.1 / 1,000$ in 2,000. They also analyzed the trend of surgical procedures for $\mathrm{BPH}$ treatment and reported a cumulative incidence of $15.2 \%$. Among the 5,671 men analyzed, TURP was the most common BPH-related prostatic surgery performed (13.4\%). Our cohort also showed TURP to be the most common surgical treatment; however, HoLEP showed an increasing trend every year.

Due to the risk associated with surgical procedures for $\mathrm{BPH}$, the use of $\alpha$-blockers increased compared to that of 5-ARIs (hazards ratio, 1.52; 95\% confidence interval [CI], 1.24-1.87). Malaeb et al. [11] analyzed the surgery trends for BPH from 2000 to 2008 using data from the Medicare carrier file. The surgical treatment pattern of $\mathrm{BPH}$ showed a reducing trend from 126,067 in 2005 to 101,195 in 2008 . A diametrical relationship was observed between the medication and surgical treatment pattens of BPH in various countries. In the analysis of patients who only received medications for $\mathrm{BPH}$ treatment, the use of $\alpha$-blockers only $(78 \%, n=518)$ was the most common, followed by use of 5-ARIs only $(9 \%, n=52)$ and a combination of both medications $(13 \%, \mathrm{n}=53)$ [11]. In our study, a similar pattern was observed with pharmacotherapy used most popularly for $\mathrm{BPH}$ treatment, and the use of $\alpha$-blockers only was the most common medication trend.

Izard et al. [12] reported a declining pattern of surgical treatment for BPH from 1988 to 1998 in Canada.

Vela-Navarrete et al. [13] analyzed the medical records of patients undergoing surgical treatment for BPH from 1992 to 2002 in Spain and reported a decrease of over 17.6\% in the number of surgical treatments performed in BPH cases. Moreover, pharmacotherapy showed an increasing trend from $46 \%$ in 1992 to $82 \%$ in $2002(\mathrm{P}<0.01)$ for $\mathrm{BPH}$ treatment, and the most common drug therapy used was $\alpha$-blockers only (79\%). Cindolo et al. [14] analyzed data from administrative and med- 
ical databases in Italy. They analyzed data on approximately 100,000 men aged $>40$ years over a period of 5 years. In this report, the medication trend for BPH increased by $43 \%$. Moreover, almost $25 \%$ increase in the use of combination medication was observed in men aged $>75$ years. The contribution of 5 -ARIs was greater in combination therapy than $a$-blockers (49\% vs. $41 \%$ ).

Since the introduction of 5-ARIs, the treatment trend has been changed to reinforce pharmacotherapy as the first treatment option [15].

In South Korea, Choi et al. [16] analyzed the BPH treatment patterns between 1985 and 2009. They categorized the 3 analysis periods as group 1 (1985-1989), group 2 (1995-1999), and group 3 (2005-2009). The comorbidities in their cohort increased over time, and this finding was different from that of a previous study. Elkoushy et al. [17] reported the variety in the characteristics of patients who underwent surgical treatment with laser for $\mathrm{BPH}$. They categorized patients based on when the surgery was performed as follows: before April 2004, between April 2004 and March 2009, and from April 2009 to August 2014. They found that patients who underwent surgical treatment were older and had more comorbidities than those who underwent pharmacotherapy.

However, other studies showed different trends $[18,19]$. Borth et al. [19] evaluated the BPH treatment trends in patients during different periods.

Mayer et al. [18] analyzed the data from randomized clinical trials on surgical treatment performed for BPH between 1997 and 2007. They analyzed 3,470 patients from 67 studies on characteristics and surgical outcomes of $\mathrm{BPH}$ patients, and there was no significant difference in age over time.

He et al. [20] reported that AUR negatively affected the outcomes in patients undergoing surgical treatment for $\mathrm{BPH}$. Among the patients undergoing bipolar TURP, those with preoperative AUR showed longer duration of hospitalization $(\geq 90$ hospital days) than those without preoperative AUR (10.7 \pm 0.9 days vs. $6.4 \pm 0.9$ days, $\mathrm{P}<0.001)$. Reich et al. [21] found that preoperative AUR also affected the risk of postoperative voiding failure (12.0\% vs. $3.5 \%$; odds ratio, 3.78 ; $95 \%$ CI, $3.20-4.45$ ). Djavan et al. [22] suggested that the high-risk group of treatment failure could be defined by chronic urinary retention (CUR) $>1,500 \mathrm{~m}$ or age $\geq 80$ years in men. Negro et al. [23] showed that the high-pressure CUR group showed poor clinical outcomes with respect to the International Prostate Symptom Score and postvoid residue (PVR).
As mentioned previously, delayed treatment could lead to increased risk of treatment failure. A previous study suggested that specific patient groups were at a high risk of progression when they were treated using only long-term medications without surgical treatment [24].

We found that pharmacotherapy only was the most common treatment trend for $\mathrm{BPH}$. In this situation, some patients may show negative effects due to long-term pharmacological treatment. Long-term pharmacological treatment may mask asymptomatic progressive detrusor impairment, leading to gradual rise in PVR [25].

Blatt et al. [26] evaluated the relationship between detrusor variables, such as muscle cell size, muscle cell shape, and collagenosis, and the risk of postoperative voiding failure. Previous reports have suggested strategizing optimal treatments in patients with high risk of progression and using only medication treatment [27]. It is unclear in which patient group would surgical treatment be the optimal treatment.

With better insight into the key factors causing urinary complications, urologists can determine the most appropriate treatment method or follow-up depending on individual risks or progress [28]. In our study, TURP was the most common surgical treatment, but HoLEP showed an increasing pattern as the surgical treatment for $\mathrm{BPH}$. Our results reviewed the treatment pattern of $\mathrm{BPH}$ and showed that pharmacotherapy was the most common treatment for $\mathrm{BPH}$, especially only a-blocker therapy.

Our study has some limitations. First, it is a potential bias to reflect actual non-BPH such as urinary stone cases. However, it is not major portion of $\mathrm{BPH}$ code, $\mathrm{BPH}$ code without $\alpha$-blocker is larger than BPH code with $\alpha$-blocker. And it is not all of urinary stone patients with BPH code with $\alpha$-blocker. Second, our data set did not cover 50s years old patients with $\mathrm{BPH}$. There are many patients with $\mathrm{BPH}$ in 50s years old. We've chosen $\mathrm{HI}-$ RA-APS due to major portion of BPH deviating older men.

In conclusion, the prevalence of $\mathrm{BPH}$ in men aged $>60$ years in South Korea showed an increasing trend in from 2012 to 2016. The major treatment method for BPH was drug therapy, and the use of $\alpha$-blockers only was the most popular treatment pattern observed in Korea. Although TRUP was the most popular surgical method used for BPH previously, its trend showed a relative reduction during the studied period, whereas HoLEP showed an increasing pattern as the surgical treatment for $\mathrm{BPH}$ in South Korea. Thus, the surgical treatment pattern of BPH has changed from TURP to HoLEP. 


\section{ACKNOWLEDGEMENTS}

The analysis of this study was performed by the Hanmi Big Data Statistical team.

\section{AUTHOR CONTRIBUTION STATEMENT}

- Conceptualization: JKJ

- Data curation: JKJ

- Formal analysis: $J K J$

- Funding acquisition: HSM

- Methodology: JKJ

- Project administration: JKJ

- Visualization: $J K J$

-Writing-original draft: JKJ

- Writing-review \& editing: SHS, KSK, HSM

\section{ORCID}

$\begin{array}{ll}\text { Jung Ki Jo } & 0000-0002-6080-7493 \\ \text { Sung Ho Shinn } & 0000-0001-9539-7941 \\ \text { Kyu Shik Kim } & 0000-0001-8755-0774 \\ \text { Hong Sang Moon } & 0000-0003-2101-1019\end{array}$

\section{REFERENCES}

1. Irwin DE, Milsom I, Hunskaar S, Reilly K, Kopp Z, Herschorn S, et al. Population-based survey of urinary incontinence, overactive bladder, and other lower urinary tract symptoms in five countries: results of the EPIC study. Eur Urol 2006;50:1306-14.

2. Suh J, Oh SJ, Cho SY. Comprehensive review of effective application of questionnaires for clinical research on lower urinary tract symptoms with translation and cultural adaptation to the Korean language. Int Neurourol J 2020;24:313-23.

3. Gratzke C, Bachmann A, Descazeaud A, Drake MJ, Madersbacher $S$, Mamoulakis $C$, et al. EAU guidelines on the assessment of nonneurogenic male lower urinary tract symptoms including benign prostatic obstruction. Eur Urol 2015;67:1099-109.

4. Jeon BJ, Chung H, Bae JH, Jung H, Lee JG, Choi H. Analysis of present status for surgery of benign prostatic hyperplasia in Korea using nationwide healthcare system data. Int Neurourol J 2019;23: 22-9.

5. Na R, Helfand BT, Chen H, Conran CA, Crawford SE, Hayward SW, et al. A genetic variant near GATA3 implicated in inherited susceptibility and etiology of benign prostatic hyperplasia (BPH) and lower urinary tract symptoms (LUTS). Prostate 2017;77:121320.

6. Wilson JD. The pathogenesis of benign prostatic hyperplasia. Am J Med 1980;68:745-56.

7. Jeong WS, Choi HY, Nam JW, Kim SA, Choi BY, Moon HS, et al. Men with severe lower urinary tract symptoms are at increased risk of depression. Int Neurourol J 2015;19:286-92.

8. Jo JK, Kim KS, Nam JW, Choi BY, Moon HS. Sociodemographic factors related to lower urinary tract symptoms in men: a Korean community health survey. Int Neurourol J 2017;21:143-51.

9. Kim KS, Jo JK, Lee JA, Choi BY, Moon HS. Do lifestyle factors affect lower urinary tract symptoms? Results from the Korean community health survey. Int Neurourol J 2019;23:125-35.

10. Souverein PC, Erkens JA, de la Rosette JJ, Leufkens HG, Herings RM. Drug treatment of benign prostatic hyperplasia and hospital admission for BPH-related surgery. Eur Urol 2003;43:528-34.

11. Malaeb BS, Yu X, McBean AM, Elliott SP. National trends in surgical therapy for benign prostatic hyperplasia in the United States (2000-2008). Urology 2012;79:1111-6.

12. Izard J, Nickel JC. Impact of medical therapy on transurethral resection of the prostate: two decades of change. BJU Int 2011;108: 89-93.

13. Vela-Navarrete R, Gonzalez-Enguita C, Garcia-Cardoso JV, Manzarbeitia F, Sarasa-Corral JL, Granizo JJ. The impact of medical therapy on surgery for benign prostatic hyperplasia: a study comparing changes in a decade (1992-2002). BJU Int 2005;96:1045-8.

14. Cindolo L, Pirozzi L, Fanizza C, Romero M, Sountoulides P, Roehrborn CG, et al. Actual medical management of lower urinary tract symptoms related to benign prostatic hyperplasia: temporal trends of prescription and hospitalization rates over 5 years in a large population of Italian men. Int Urol Nephrol 2014;46:695-701.

15. Oelke M, Bachmann A, Descazeaud A, Emberton M, Gravas S, Michel MC, et al. EAU guidelines on the treatment and follow-up of non-neurogenic male lower urinary tract symptoms including benign prostatic obstruction. Eur Urol 2013;64:118-40.

16. Choi SY, Kim TH, Myung SC, Moon YT, Kim KD, Kim YS, et al. Impact of changing trends in medical therapy on surgery for benign prostatic hyperplasia over two decades. Korean J Urol 2012; 53:23-8.

17. Elkoushy MA, Elshal AM, Elhilali MM. Changing patients' profile presenting for surgical management of benign prostatic hyperplasia over the past 16 years: a single-centre perspective. Can Urol Assoc J 2015;9:372-8.

18. Mayer EK, Kroeze SG, Chopra S, Bottle A, Patel A. Examining the 'gold standard': a comparative critical analysis of three consecutive de- 
cades of monopolar transurethral resection of the prostate (TURP) outcomes. BJU Int 2012;110:1595-601.

19. Borth CS, Beiko DT, Nickel JC. Impact of medical therapy on transurethral resection of the prostate: a decade of change. Urology 2001;57:1082-5; discussion 5-6.

20. He LY, Zhang YC, He JL, Li LX, Wang Y, Tang J, et al. The effect of immediate surgical bipolar plasmakinetic transurethral resection of the prostate on prostatic hyperplasia with acute urinary retention. Asian J Androl 2016;18:134-9.

21. Reich O, Gratzke C, Bachmann A, Seitz M, Schlenker B, Hermanek $\mathrm{P}$, et al. Morbidity, mortality and early outcome of transurethral resection of the prostate: a prospective multicenter evaluation of 10 , 654 patients. J Urol 2008;180:246-9.

22. Djavan B, Madersbacher S, Klingler C, Marberger M. Urodynamic assessment of patients with acute urinary retention: is treatment failure after prostatectomy predictable? J Urol 1997;158:1829-33.

23. Negro CL, Muir GH. Chronic urinary retention in men: how we define it, and how does it affect treatment outcome. BJU Int 2012; 110:1590-4.

24. Presicce F, De Nunzio C, Tubaro A. Can long-term LUTS/BPH pharmacological treatment alter the outcomes of surgical intervention? Curr Urol Rep 2017;18:72.

25. Van Koeveringe GA, Rademakers KL. Factors impacting bladder underactivity and clinical implications. Minerva Urol Nefrol 2015; 67:139-48.

26. Blatt AH, Brammah S, Tse V, Chan L. Transurethral prostate resection in patients with hypocontractile detrusor-what is the predictive value of ultrastructural detrusor changes? J Urol 2012;188:2294-9.

27. Oelke M, Kirschner-Hermanns R, Thiruchelvam N, Heesakkers J. Can we identify men who will have complications from benign prostatic obstruction (BPO)? ICI-RS 2011. Neurourol Urodyn 2012; 31:322-6

28. Tubaro A, De Nunzio C, Puccini F, Presicce F. The evolving picture of lower urinary tract symptom management. Eur Urol 2015;67:271-2. 